\title{
Fuzzy Logic Partition-Based Call Admission Control for Mobile WiMAX
}

\author{
D. S. Shu'aibu, S. K. Syed Yusof, N. Fisal, S. H. S. Ariffin, R. A. Rashid, \\ N. M. Abdul Latiff, and Y. S. Baguda
}

Faculty of Electrical Engineering, Universiti Teknologi Malaysia, Skudai, 81310 Johor, Malaysia

Correspondence should be addressed to D. S. Shu'aibu, dahiru.75@gmail.com

Received 2 February 2011; Accepted 11 April 2011

Academic Editor: G. Giambene

Copyright (C) 2011 D. S. Shu'aibu et al. This is an open access article distributed under the Creative Commons Attribution License, which permits unrestricted use, distribution, and reproduction in any medium, provided the original work is properly cited.

\begin{abstract}
The unpredictable nature of the wireless network and exponential growth in traffics with different quality of service requirements has led hardware complexity to escalate. In order to effectively control and manage the network traffics, there is a need for intelligent call admission control (CAC) in admitting traffics into the wireless network that provides necessary quality of service. In this paper, we propose a fuzzy logic partition-based call admission control (FZ CAC). The scheme primarily partitions the total link bandwidth into three which corresponds to constant bit rate (CBR), variable bit rate (VBR) and handover (HO) services. The fuzzy logic admission control scheme was implemented in the HO portion to intelligently keep dropping probability as low as possible based on the available bandwidth. Simulation results showed that the proposed approach outperformed both partition-based CAC (PB CAC) and conventional bandwidth allocation CAC (CB CAC).
\end{abstract}

\section{Introduction}

WiMAX is one of the recent broadband wireless communication technologies, which supports five different scheduling services. It has a large coverage area per base station which can be up to 30 miles in radius [1]. To increase the coverage and throughput, relay stations were introduced by IEEE (WiMAX Forum) [1]. This eventually increases the chance of more traffic to compete for the limited bandwidth. To handle for all these traffics, WiMAX supports adaptive modulation and coding, such that the distance between the subscriber station (SS) and the base station (BS) determines the type of modulation to be used $[1,2]$.

In WiMAX, the minimum resource that can be allocated to SS is a slot. The definition of a slot depends on the type of permutation used. Four types of permutations are used in WiMAX: partial usage subchannelization (PUSC), full usage subchannelization FUSC, adaptive modulation and coding (AMC) and tile usage subchannelization (TUSC) [1]. Bandwidth request by SS is either by unicast polling, multicast group polling piggyback or through ranging channels [1]. When the BS responds, the bandwidth can be granted by grant per subscriber station (GPSS) or by grant per connection (GPC). In GPSS, the BS grants a bandwidth on per subscriber basis such that, all its connection is treated as a block. Hence, there is need for a local scheduler at SS to control the service order and its QoS. In GPC mode, the bandwidth is granted per connection packet. The request of this kind is by piggyback.

BS can communicate in either point to multipoint (PMP) or mesh mode. In PMP, the BS controls all the communication within its own coverage. In mesh mode, the SS can form a mesh connection without the need of BS. The five scheduling services supported in WiMAX are Unsolicited Grant Service (UGS), real time polling service (rtPs), extended real-time polling service (ertPs), nonrealtime polling service (nrtPs), and best effort (BE) [1]. The UGS is a real-time application service with CBR. Each packet has a fixed size and transmitted at equal interval of time. The rtPs is another real-time application service with VBR where each packet may have different sizes transmitted at unequal interval of time. The ertPs is similar to UGS except that there is silence suppression associated with it. The nrtPs is an application which does not require real time; 
the application has VBR with packets having different size transmitted at unequal interval of time. Lastly BE, is nonreal time application requires the minimum bit error rate in transmission.

In this paper, we present efficient call admission control (CAC) which is partitioned based on fuzzy logic control of the handover $(\mathrm{HO})$ partition. Our main focus is to reduce the dropping probability and increase the service flow acceptance. It uses GPSS and PMP mode. The paper is organized as follows: Section 2 focuses on related work, Section 3 deals with the system model, Section 4 discusses the fuzzy logic controller. The proposed CAC is presented in Section 5. Simulation parameters are presented in Sections 6 and Section 7 concludes the paper.

\section{Related Work}

The IEEE802.16 standard defines five scheduling classes, but it does not provide any information about the standard algorithms that should be used to provide QoS to the service flows (SFs). CAC plays an important role especially when combined with scheduling service, since they are meant to manage and guarantee the QoS requirements. A single CACs algorithm cannot guarantee the QoS without the support of scheduling algorithm and vice versa [3]. Although these algorithms can be designed separately, each has to be efficient in managing the network resources. The CAC handles the performance of the link bandwidth while the scheduling handles other QoS parameters like delay, jitter, latency, and slot allocation.

Several research works have been conducted in order to provide QoS in IEEE 802.16 networks [3-10]. In [3], QoS scheduling architecture for the MAC protocol in 802.16 networks was presented. It is based on dynamic bandwidth allocation in the CAC and service flow (SF) priority in the scheduling algorithm. The bandwidth grant was based on GPSS mode, but no simulation result was presented, hence the performance of this architecture was not evaluated. In [4], GPC mode for bandwidth allocation was adopted and designed for an uplink scheduling architecture in IEEE802.16 to support diverse QoS such as bandwidth and delay. It is a centralized approach in point to multipoint mode; all scheduling decisions are achieved through the BS. There is no simulation result to demonstrate effectiveness of this architecture.

In [5] a simple CAC algorithm was presented, the bandwidth allocation algorithm proposed was hierarchical structure for downlink which decides whether QoS for a particular connection can be satisfied at the BS or not. The approach used conventional bandwidth allocation (CB CAC). A similar work with adaptive bandwidth allocation was considered in [6] which are applied to cellular network. In [7], a fuzzy logic was used in order to reduce the dropping probability of the mobile cellular network. The problem of mobility management was addressed in [8], two machine learning techniques were used, fuzzy logic and particle swarm optimization (PSO) which enhanced the accuracy of link break prediction and congestion control in vehicular ad hoc networks. The authors in [9] improved the work of [5] by considering packets' delay. The architecture is called QoS CAC and GPC mode is employed. It is primarily aimed at reducing packet delay and bandwidth guarantee for various applications while achieving high system utilization with fewer figures of merit. Flow acceptance was reduced compared to work by [5].

To improve the service flow acceptance and service level differentiation in [5], a partition-based architecture called partition-based $\mathrm{CAC}$ (PB-CAC) is proposed in this paper. PB-CAC is an improvement of work in [10]. In both $[5,10]$, the handover traffics have not been given special considerations. HO service flows are very important and unavoidable in WiMAX. Prematurely terminating of a service flow from the network leads to dropping the service flow and it mostly happens during handover traffics. Blocking a service flow is to deny the service flow from using the network. In order to intelligently reduce both blocking of service flows and dropping of $\mathrm{HO}$ services we present a CAC that uses fuzzy logic scheme. The proposed fuzzy logic call admission control (FZ CAC) is simple and efficient; we developed the algorithms in $[5,10]$ and compared them with our developed system. The following section describes the mathematical model of the system.

\section{System Model}

The system model has one central BS and neighboring base station that generates handover traffics. SSs are uniformly distributed around the coverage area of the central BS and the neighboring BS. Hilly terrain environment with moderate to heavy tree density which reflects category A of Erceg model [1] has been used for the simulation. The total path loss, $\mathrm{Pl}$ between SSs and BS is a function of distance and can be expressed mathematically as shown below:

$$
\begin{aligned}
P_{\text {loss }}= & 20 \log _{10}\left(\frac{4 \pi d_{0}}{\lambda}\right)+10 \gamma \log _{10}\left(\frac{d}{d_{0}}\right)+6 \log _{10}\left(\frac{f}{2000}\right) \\
& -10.8 \log _{10}\left(\frac{h}{2}\right)+s, \quad \text { for } d>d_{0},
\end{aligned}
$$

where $\lambda$ is wavelength, $d_{o}$ is the reference distance $(100 \mathrm{~m})$, $\gamma$ is path loss exponent, $d$ is the distance of SS from the BS which is greater than $d_{0}, f$ is the transmission frequency, $h$ is the BS antenna height and $s$ is the shadowing factor. When the total path loss is computed using (1), the signal to noise ratio, SNR can be obtained using the following equation [11]:

$$
\mathrm{SNR}=\frac{\varepsilon \theta \vartheta}{\mathrm{Pl} * \psi},
$$

where $\varepsilon$ is transmitted power from the BS, $\theta$ is BS antenna gain, $\vartheta$ is SS antenna gain, $\psi$ is the receiver sensitivity which is given by

$$
\psi=f\left(\beta, \tau, \text { Noise, } \ell, K, E_{c}\right),
$$

where $\beta$ is the receiver implementation loss, $\tau$ is Boltzmann constant, $\ell$ is the modulated symbol rate, $K$ is environmental 
TABLE 1: Receiver SNR in (dB).

\begin{tabular}{lccc}
\hline S/N & Modulation & Coding rate & SNR $(\mathrm{dB})$ \\
\hline \multirow{2}{*}{1} & \multirow{2}{*}{ QPSK } & $1 / 2$ & 5.0 \\
& & $3 / 4$ & 8.0 \\
\hline \multirow{2}{*}{2} & \multirow{2}{*}{ 16-QAM } & $1 / 2$ & 10.5 \\
& & $3 / 4$ & 14.0 \\
\hline \multirow{3}{*}{3} & \multirow{2}{*}{ 64-QAM } & $1 / 2$ & 16.0 \\
& & $2 / 3$ & 18.0 \\
& & $3 / 4$ & 20.0 \\
\hline
\end{tabular}

temperature in Kelvin and $E_{c}$ is the received energy per modulated symbol [1]. When the SS distance to BS is known, the total path loss is computed which is used to obtain the SNR value in (2). The SNR value in (2) is then used in choosing the required modulation scheme and coding rate as shown in Table 1 [2]. For example, if the SNR value evaluated from (2) in $\mathrm{dB}$ is between $5 \mathrm{~dB}$ and $8 \mathrm{~dB}$ the modulation scheme chosen will be QPSK with 1/2 coding rate and no modulation will be assign for SNR less than $5 \mathrm{~dB}$.

When SS bit rate and SNR are known, the required number of subcarriers and the number of slots can be obtained. The total number of slot per frame depends on the permutation used on the frame [1]. A slot is one subchannel by OFDMA symbol, for PUSC permutation, a slot (in the downlink) is one subchannel by two OFDMA symbols therefore the total number of slots $\left(T_{s}\right)$ on one PUSC frame is given by

$$
T_{s}=\frac{s_{c} * f_{d}}{2},
$$

where $s_{c}$ is the total number of subchannel, $f_{d}$, is the number of OFDMA symbols in the downlink. Thus the total number of slots $\left(T_{0}\right)$ in one second is

$$
T_{0}=\frac{T_{s}}{T_{d}},
$$

where $T_{d}$ is the frame duration in a second. Based on the SNR value at a given time, one subcarrier $\left(J_{s}\right)$ in a slot can give a data rate given as

$$
J_{s}=f_{d} * c_{r} * \frac{\log _{2}(m)}{T_{d}},
$$

where $c_{r}$ is the coding rate and $\log (m)$ is the modulated symbol. For traffic with bit rate $\Omega_{t}$ bps, the number of subcarriers $\left(N_{s}\right)$ required in one second for the bit rate is given as

$$
N_{s}=\text { floor }\left(\frac{\Omega_{t}}{J_{s}}\right) \text {. }
$$

And hence the number of slots required to satisfy the bit rate is,

$$
\mathrm{sl}=\operatorname{ceil}\left(\frac{N_{s}}{K_{s}}\right),
$$

where $K_{s}$ is the number of subcarriers per slot. Each slot $\left(S_{r}\right)$ is upper-bounded by

$$
S_{r}=K_{s} * c_{r} * \log _{2}(m) .
$$

Hence the total network capacity (NC) is thus given by (5) and (9) as

$$
\mathrm{NC}=T_{s} * K_{s} * c_{r} * \frac{\log _{2}(m)}{T_{d}} .
$$

The total number of bits in one frame is thus

$$
T_{f}=\mathrm{NC} * T_{d},
$$

where $T_{d}$, is in seconds.

The total downlink network capacity is partitioned into 3 portions, CBR, VBR, and the handover traffic (HO). Each portion is some percentage of the total capacity. Both new traffic and handover traffic follow independent Poisson distribution therefore the bandwidth of the $\mathrm{HO}$ portion slightly adjusted using fuzzy logic to intelligently avoid blockage. This reduces the bandwidth of the CBR portion or VBR portion depending on which one has a larger bandwidth at the time of using fuzzy logic controller. The fuzzy logic controller is activated when there is call and the remaining bandwidth of $\mathrm{HO}$ is less than the minimum sustainable traffic rate. To approximately derive the dropping probability $\left(P_{d}\right)$, we use the ratio as $P_{d}=x / y$, where $x$ is number of unsuccessful handover and $y$ is number of handover traffics. Similarly, the same approximation is used in computing blocking probability $\left(P_{b}\right)$ which is given by $J / Z$, where $J$ is the number of traffics blocked and $Z$ is the total number of traffics requesting for network entry.

\section{Fuzzy Logic Controller}

The CAC algorithm presented in [10] use partition approach to increase the total acceptance flow in [5]. In our developed algorithm, we use fuzzy logic control to increase the service flow acceptance by reducing both blocking and dropping probability of the handover service when compare to [5]. Fuzzy logic controller has been used quite extensively in many engineering application and system $[12,13]$. In this paper, we use fuzzy logic scheme based on Mandani fuzzy model. The model has 3 inputs: the number of call drop, available bandwidth in CBR and VBR portion with one output, and adjustment of the handover bandwidth. Triangular membership function has been used due to the fact that it is simple and easy to implement [12]. The structure of the fuzzy logic controller is shown in Figure 1.

The fuzzifier receives the 3 input variables and converts them to suitable linguistic values (input membership) needed by the inference engine. The inference receives the input membership and by using fuzzy IF-THEN rule it makes decision on the output membership. The output membership is use by the defuzzifier to generate a non fuzzy control output; that is the amount of bandwidth to be added to the current $\mathrm{HO}$ bandwidth.

4.1. Membership Function. The membership functions were chosen based on the behavior observed from the system. The number of total drop has five membership function $M($ drop $)=f\{$ VS S M B VB $\}$. The available bandwidth, 


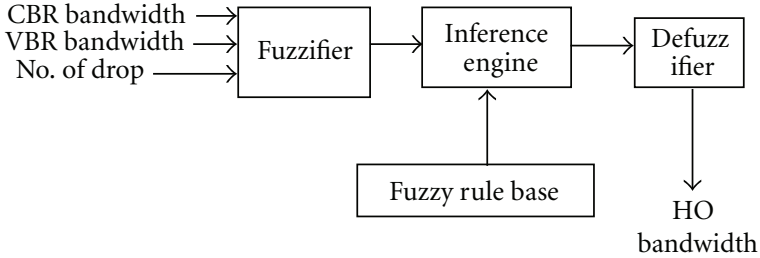

FIgURe 1: The structure of fuzzy logic controller for CAC.

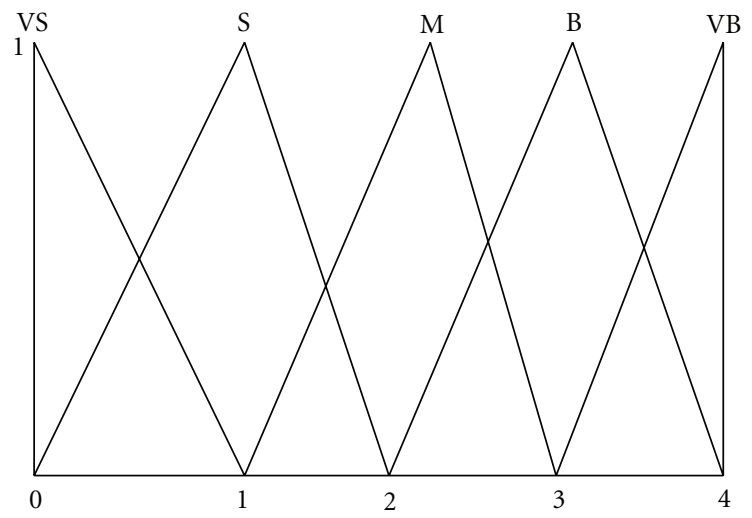

FIgURe 2: Membership function for dropping.

which is the positive difference between VBR and CBR bandwidth has five membership function $M$ (available) = $f\{$ VS $S \mathrm{M} \quad \mathrm{B} \quad \mathrm{VB}\}$. These are the inputs variable with their membership function. The membership functions of the output were carefully selected so that both the drop and block can be reduced, due to the fact that increase in traffics portion has detrimental impact on other traffic's portion. Eight membership functions were used at the output. $M$ (adjust $)=f\{$ Z VS S SM M MB B VB $\}$. Figures 2,3 , and 4 shows the membership functions for the inputs and output linguistic parameters.

4.2. Fuzzy Rule Based. Each of the two variables at the input of fuzzy controller has 5 membership functions. Therefore from the input, we have 25 different combinations, each one is control by one IF THEN rule. Hence the fuzzy logic uses 25 IF THEN rules, in which only one of the rules is triggered whenever there is a dropping of an existing service flow. This is the strategy we used to increase the handover bandwidth portion. In this paper, the available bandwidth is scaled by a factor $R$, where $R$ is defined as the maximum rate of the CBR traffics and the value of $R$ is set to $64 \mathrm{kbps}$. Hence, the available bandwidth is expressed in the form $n R$, where $n$ is the number of times when the available bandwidth is more than $R$. The membership's function of call drop is shown in Figure 2. Based on the IF THEN rule, the output bandwidth of the $\mathrm{HO}$ can be increased from $0 \%$ to $35 \%$. The fuzzy logic rules are shown in Table 2.

The rules in Table 2 above are carefully formed to avoid unnecessary blocking and minimize dropping of handover service. Rule 13 from Table 2 means that if the total drop is medium and maximum available bandwidth is medium then

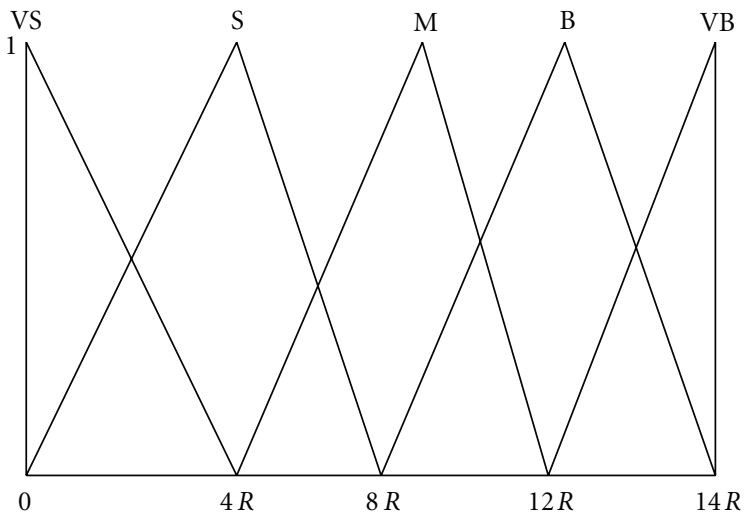

FIgURE 3: Membership function for available bandwidth.

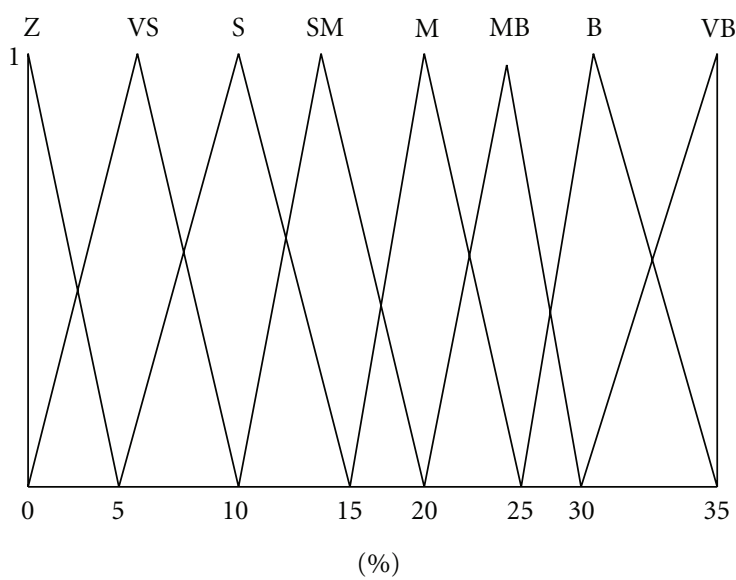

Figure 4: Membership function for adjusting the bandwidth. Definition of the membership function is as follow: Z: zero; VS: very small; S: small; SM: small medium; M: medium; MB: medium big; B: big; VB: very big.

fuzzy logic should adjust the bandwidth to small medium. The fuzzy logic in this case will reduce the bandwidth of CBR or VBR by $15 \%$ to $20 \%$ whichever one is high and increase the same amount to HO bandwidth. The mathematical expression is as follows, if we let

$$
\begin{gathered}
h=C-V, \quad h= \begin{cases}1, & \text { if } C>V, \\
0, & \text { otherwise, }\end{cases} \\
k=C * h * \sum_{i=1}^{25} F z(i)+V * \vec{h} * \sum_{i=1}^{25} F z(i),
\end{gathered}
$$

where $C$ is the available bandwidth in CBR, $V$ is the available bandwidth in VBR $\vec{h}$ is logical not $h$ and $F z(i)$ is one of the twenty five fuzzy rules, evaluated to logic 1 if it is true. The output from the defuzzifier adjusts bandwidth of handover portion given as

$$
y=\mathrm{HO}=k * p_{0},
$$

where $p_{0}$, is one of the output membership functions express in percentage of the selected bandwidth portion and $\mathrm{HO}$ 
TABLE 2: CAC fuzzy logic controller rules.

\begin{tabular}{|c|c|c|c|}
\hline $\mathrm{S} / \mathrm{N}$ & If total call drop is & and the max bandwidth is & then adjust the bandwidth to $F z(i)$ \\
\hline 1 & very small & very small & zero \\
\hline 2 & very small & small & very small \\
\hline 3 & very small & medium & very small \\
\hline 4 & very small & big & very small \\
\hline 5 & very small & positive big & very small \\
\hline 6 & small & very small & very small \\
\hline 7 & small & small & very small \\
\hline 8 & small & medium & small \\
\hline 9 & small & big & small \\
\hline 10 & small & positive big & small medium \\
\hline 11 & medium & negative small & very small \\
\hline 12 & medium & small & small \\
\hline 13 & medium & medium & small medium \\
\hline 14 & medium & big & medium \\
\hline 15 & medium & positive big & big medium \\
\hline 16 & big & negative small & very small \\
\hline 17 & big & small & very small \\
\hline 18 & big & medium & small \\
\hline 19 & big & big & medium \\
\hline 20 & big & positive big & big medium \\
\hline 21 & very big & negative small & very small \\
\hline 22 & very big & small & small \\
\hline 23 & very big & medium & very big \\
\hline 24 & very big & big & very big \\
\hline 25 & very big & positive big & very big \\
\hline
\end{tabular}

is current available bandwidth before adjustment by fuzzy logic.

\section{Fuzzy Logic Partition-Based Call Admission Control (FZ CAC)}

In [5], the admission control scheme uses a conventional bandwidth control algorithm. It receives dynamic service addition (DSA) for new traffic request, dynamic service changes (DSC) for existing traffic to change its service flow and dynamic service delete (DSD) for existing traffic to delete its service flow or leaving the network and updated the bandwidth by using the following bandwidth control equation

$$
\mathrm{BW}=\mathrm{BW}_{\text {total }}-\sum_{n=0}^{N} \sum_{m=0}^{M} K_{\min }(n, m),
$$

where $\mathrm{BW}$ is the available bandwidth, $\mathrm{BW}_{\text {total }}$ is the total capacity of the bandwidth, $N$ is the number of service classes, such that $n$th class of service has a total of $M_{n}$ connection. $K_{\min }(n, m)$ is the minimum reserved traffic rate for $m$ th connection in the $n$th class of service flow.

Our approach partitioning the bandwidth into 3 portions, and admit all CBR traffics in CBR portion, VBR traffics in VBR portion, and handover service in HO portion. The bandwidth is updated after admitting traffic, by subtracting the required bandwidth to give QoS of the service involved from the remaining bandwidth of the portion. However, when there is a drop, fuzzy logic is implemented in the HO portion to adjust the bandwidth by borrowing from either CBR or VBR portion and update all the 3 portions before the next traffic.

The proposed algorithm (Algorithm 1) has the following parameters: for handover traffic we have handover service request (HSR), for $\mathrm{HO}$ traffic, HSR is set to 1 and zero otherwise. We defined the service flows identification (SFID) as follows: when SFID is 1 the traffic is UGS which is CBR, for SFID $=2$, the traffic is rtPs, for SFID $=3$, the traffics is nrtPs and when SFID $=4$, the traffic is BE and all are VBR. The bandwidth is allocated as follows: CBR maximum rate is allocated $(\max ), \mathrm{VBR}$ real-time average of maximum and average is allocated $((\max +$ ave $) / 2)$, for VBR nonreal-time allocated bandwidth is the average of minimum and average rate $(($ ave $+\min ) / 2)$ and for best effort is always admitted and allocated with minimum rate ( $\mathrm{min})$. The sum of all allocated bandwidth to these traffics is less than the link bandwidth.

\section{Simulation Results}

In the simulation, bandwidth is granted by grant per subscriber station (GPSS) mode so that, traffic is admitted once and can manage its QoS. The simulation parameters are 
Initialize all parameters

(1) Let the total bandwidth be $B_{T}$

(2) Partition into CBR, VBR and handover $\left(B_{C}, B_{V}, B_{H}\right)$

(3) Deploy the traffics randomly; both the new and existing

For $j=1: N \quad \% N$ is total service flows

(4) Use (1), (2), (6), (7), and (8) to admit the Service flow

(5) If HSR $=1 ; \%$ this is handover, with DSA $=1$

already \%a HSR is for handover traffics

(6) If $\max <B_{H} \& \&$ SFID $=1 ; \%$

Allocated_rate $=\max$

update $B_{H}$

Else Reject

Update total drop

Apply Fuzzy logic to adjust the $B_{H}$ End

Update $B_{H}, B_{V}$, and $B_{C}$

(7) Else If SFID = 2, repeat 6 with allocated_rate $=\max +$ ave $/ 2$

(8) Else If SFID = 3, repeat 6 with allocated_rate $=$ ave $+\min / 2$

(9) Else SFID =4, repeat 6 with allocated_rate $=\min$ END

(10) If DSC $=1 ; \%$ traffics changing services flow Restore the initial bandwidth of the service flow to $B_{H}$ Update $B_{H}$ If the current bandwidth of $B_{H}>$ the new request Allocate the bandwidth Else Reject

Update total drop

Apply Fuzzy logic to adjust the $B_{H}$ END Update $B_{H}, B_{V}$, and $B_{C}$

(11) IF DSD $=1 ; \%$ traffics deleting services flow Restore the bandwidth and update $B_{H}$ END End for

Algorithm 1: Algorithm for FZ CAC.

shown in Tables 3 and 4 . In Table 5 we have the total number of new traffics and handover service for different scenarios.

In order to critically analyse the performance of the developed scheme, three different scenarios were used in the simulation, namely, light, medium, and heavy traffics. Each scenario has some number of new service flows and existing service flows as shown in Table 5.

The traffics are uniformly distributed and requesting for network entry by sending DSA/DSC/DSD/HSR message in Poisson distribution. The algorithm in [5] called (CB-CAC) accepts traffics as long as there is enough bandwidth and does not have control on the type of traffics and no service level differentiation. The algorithm in [10] known as (PB-CAC) on the other hand divides the bandwidth into CBR portion, $\mathrm{VBR}$, and $\mathrm{HO}$ portion, and it allocates a fixed percentage to each irrespective of the nature of the arriving service flows. The service flows are accepted as long as there is enough bandwidth in their respective portion.

In the proposed scheme (FZ CAC), bandwidth is divided into $\mathrm{CBR}, \mathrm{VBR}$, and $\mathrm{HO}$ as well. It allocates bandwidth
TABle 3: Parameter settings.

\begin{tabular}{lc}
\hline Parameter & Settings \\
\hline FFT Size & 512 \\
Bandwidth & $5 \mathrm{MHz}$ \\
DL/UL ratio & $35 / 12$ \\
No. of Subchannels & 15 \\
No. of Subcarriers & 360 \\
Frame duration & $5 \mathrm{~ms}$ \\
Frame Modulation & $64-\mathrm{QAM}$ \\
Total Capacity & $12.6 \mathrm{Mbps}$ \\
Handover portion & $10 \%$ \\
CBR portion & $20 \%$ \\
SVBR portion & $70 \%$ \\
Modulation scheme & $16-\mathrm{QAM}, 64-\mathrm{QAM}$ \\
Path loss model & $\mathrm{Erceg}$ \\
Transmission frequency & $2.5 \mathrm{GHz}$ \\
Path loss exponents & 4.75 \\
Shadowing component & 8.9 \\
BS transmitting power & $43(\mathrm{dBm})$ \\
BS antenna gain & $15(\mathrm{dBi})$ \\
Receiver sensitivity & $-111.1(\mathrm{dBm})$ \\
Receiver antenna gain & $-1.0(\mathrm{~dB})$ \\
\hline
\end{tabular}

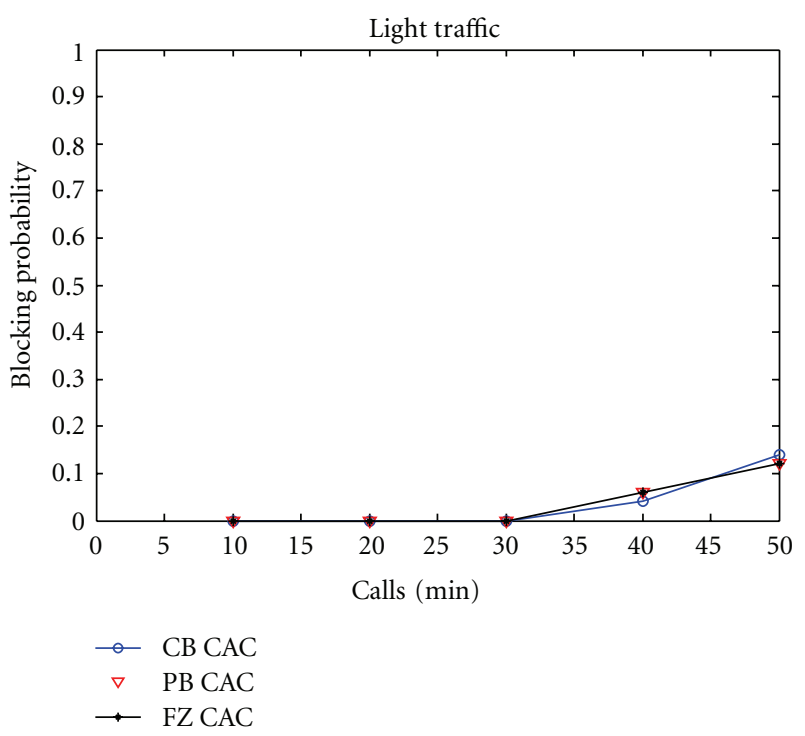

Figure 5: Blocking probability in light traffics.

Table 4: Traffic rate in Kbps.

\begin{tabular}{lcccc}
\hline Class & Type & Max & Ave & Min \\
\hline Traffic 1 & UGS & 64 & 64 & 64 \\
Traffic 2 & UGS & 20 & 20 & 20 \\
Traffic 3 & rtPs & 400 & 380 & 320 \\
Traffic 4 & rtPs & 82 & 60 & 53 \\
Traffic 5 & nrtPs & 130 & 126 & 110 \\
Traffic 6 & nrtPs & 2000 & 1870 & 1600 \\
\hline
\end{tabular}




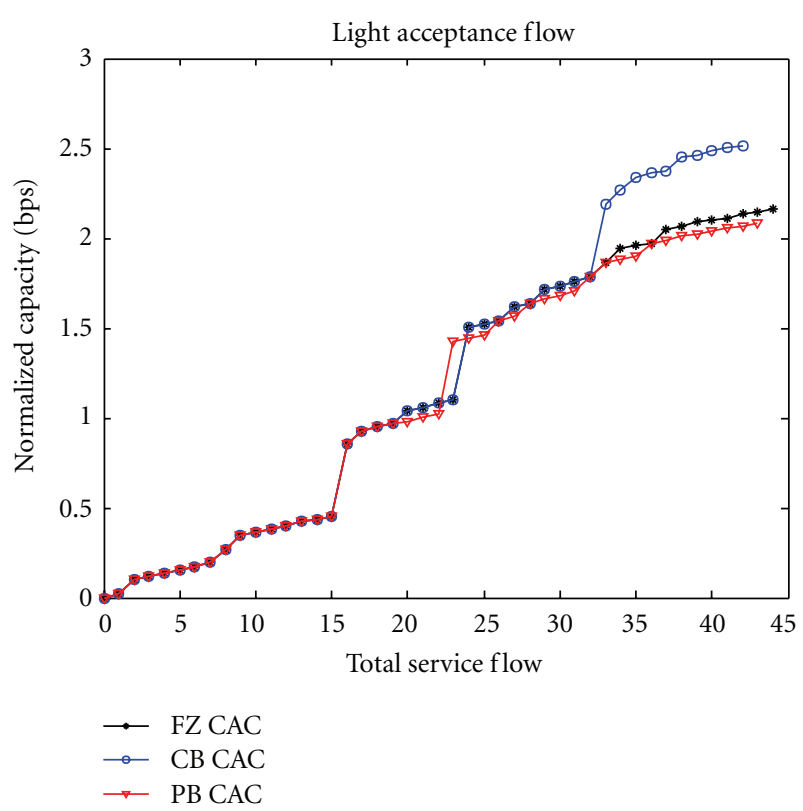

FIgURE 6: Normalized capacity of the total acceptance flow.

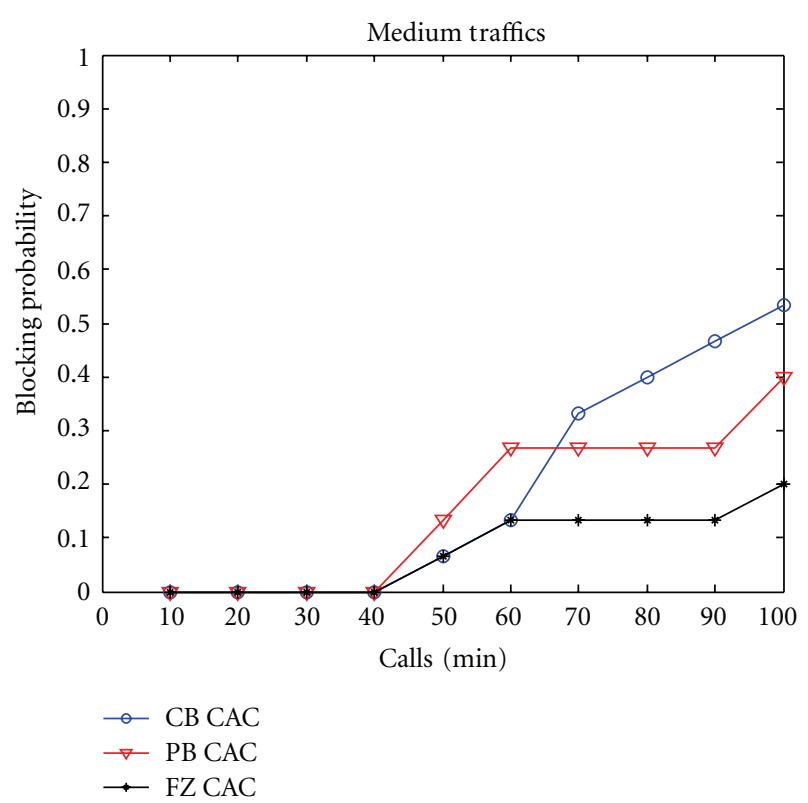

Figure 7: Dropping probability in medium traffics.

TABLE 5: Scenario.

\begin{tabular}{lcc}
\hline Scenario & New SF & Existing SF \\
\hline Light traffics & 40 & 10 \\
Medium traffics & 85 & 15 \\
Heavy traffics & 130 & 20 \\
\hline
\end{tabular}

to traffics with dynamics percentage to each portion, using initial value $10 \%, 20 \%$, and $70 \%$ for $\mathrm{HO}, \mathrm{CBR}$, and VBR, respectively. Service flows are admitted as long as there is enough bandwidth in their respective portion. We classified

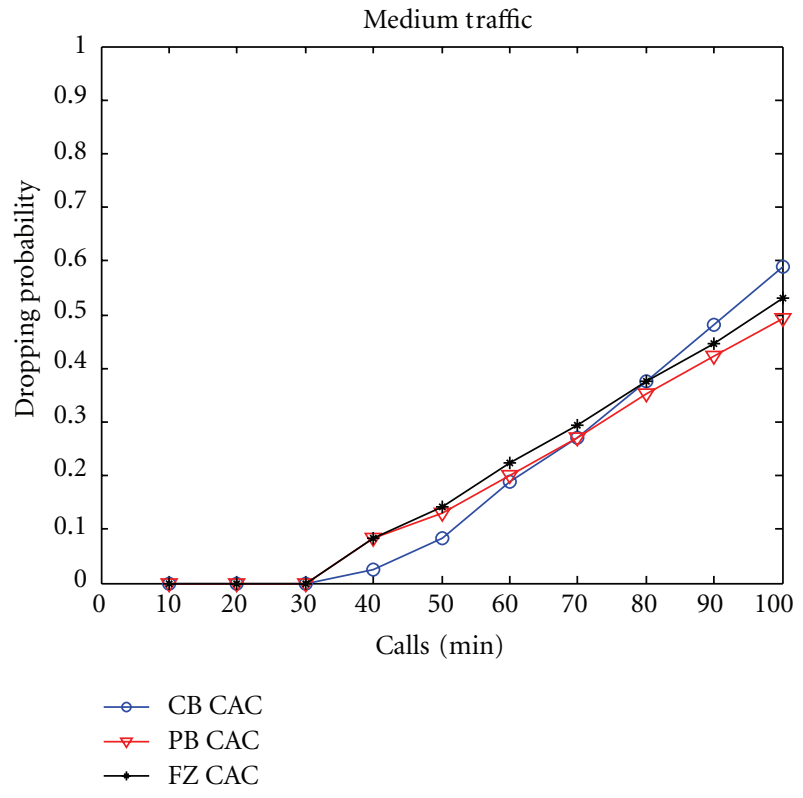

FIGURE 8: Blocking probability in medium traffics.

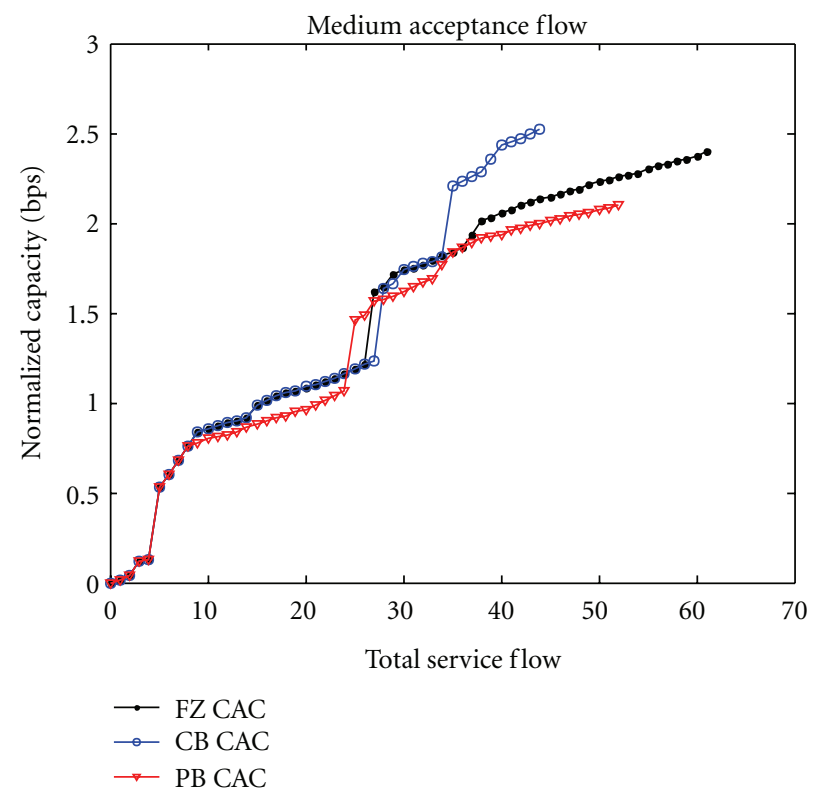

FIgURE 9: Normalized capacity of the total acceptance flow in medium traffics.

the scenario as either light traffics with few service flows, medium traffics with medium number of service flow and heavy traffics when too many SSs are involved. The following section explain the scenario in details.

6.1. Light Traffics. In this scenario, we have 40 new service flows and 10 existing or handover service flows are used. The traffics are uniformly distributed around the coverage area. It was found out that in this scenario, all the three approaches have accepted all handover service flows. However, not all new traffics were admitted as shown in Figure 6, and the 


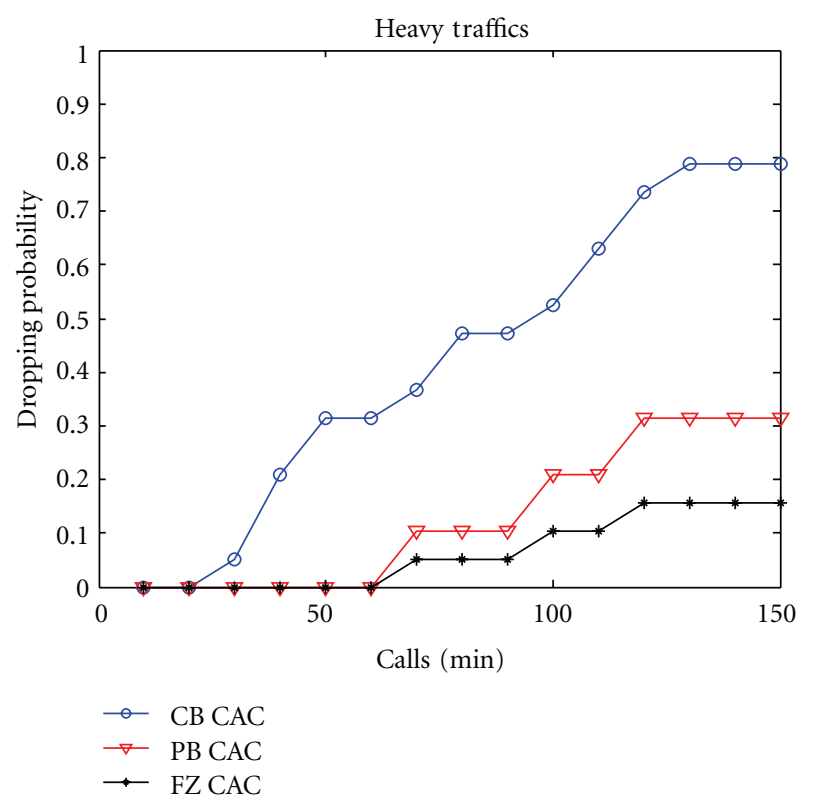

FIGURE 10: Dropping probability in heavy traffics.

blocking probability performance is shown in Figure 5. The blocking probability is evaluated every 5 calls $/ \mathrm{min}$. The blocking probability for FZ CAC and PB CAC in this scenario is the same but lower than CB CAC. The total service flow acceptance is shown in Figure 6, it can be seen that FZ CAC has about $2.27 \%$ improvement than $\mathrm{PB}$ CAC and $\mathrm{CB}$ CAC in light traffics.

6.2. Medium Traffics. In this scenario, 85 new service flows and 15 handover service flows are employed. It can be seen from Figure 7 that FZ CAC has much lower dropping probability than the two schemes. It has a lower blocking probability as shown in Figure 8 than CB CAC. Also it can be seen from Figure 9, FZ CAC has higher service acceptance flow when compared with other approaches. With reference to $\mathrm{CB}$ CAC at service flow index $45, \mathrm{FZ}$ CAC has $17 \%$ improvement while $\mathrm{PB} \mathrm{CAC}$ has $8 \%$ improvement over $\mathrm{CB}$ CAC.

6.3. Heavy Traffics. In heavy traffic, we use 130 new service flows with 20 handover traffics. It can be seen that the dropping in FZ CAC is much lower in this scenario the network is oversubscribed as shown in Figure 10. The bandwidth approached saturation level. The blocking probability also is low in FZ CAC compared to CB CAC as in Figure 11. The acceptance flows is shown in Figure 12. FZ CAC has improved the acceptance flow by $10.6 \%$ and PB CAC improved the acceptance flows by only $6 \%$ with reference to $\mathrm{CB} C A C$ at service flow index number 53 .

6.4. FZ CAC Performances. The performance of FZ CAC scheme is shown in Figure 13, which is the normalized bandwidth capacity of $10 \%, 20 \%$, and $70 \%$ initially assigned to $\mathrm{HO}, \mathrm{CBR}$, and VBR, respectively. The degradation of the

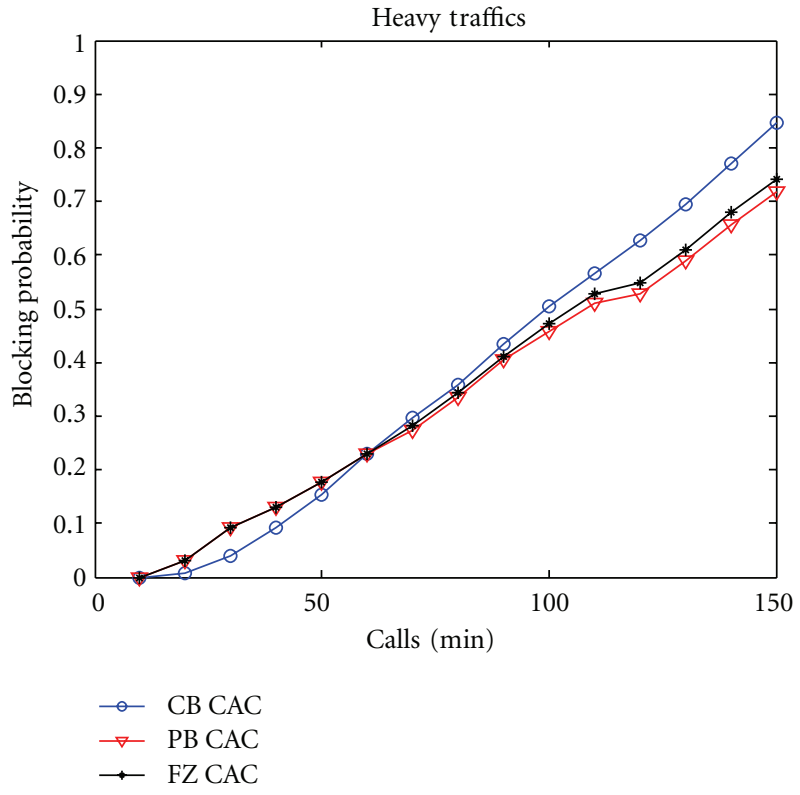

FIGURE 11: Blocking probability in heavy traffics.

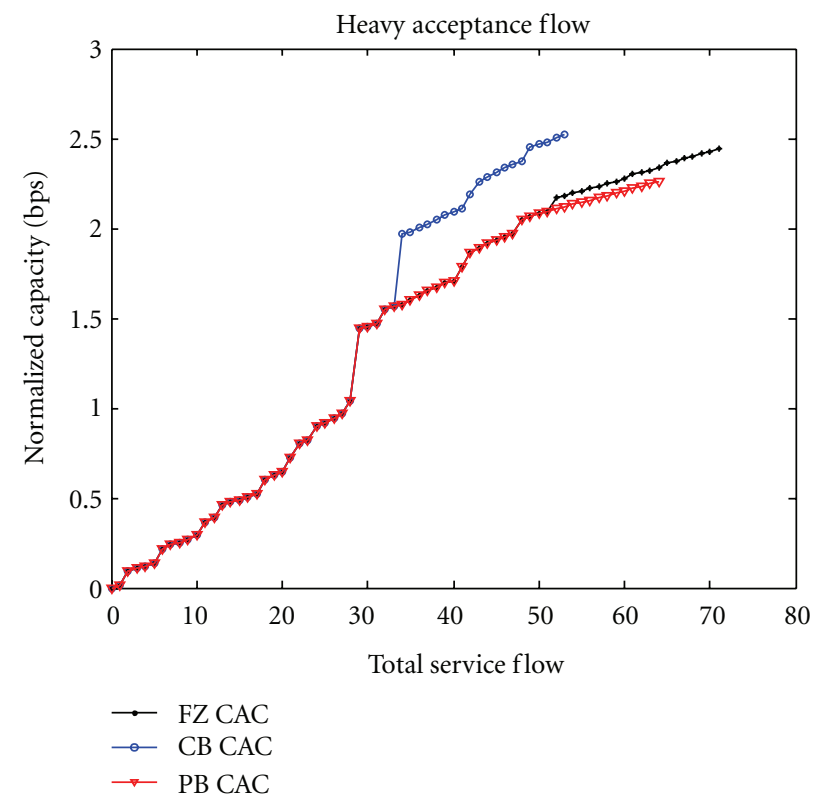

Figure 12: Normalized capacity of the total acceptance flow in heavy traffics.

normalized bandwidth can be seen as the acceptance service flow increases in all the three portions. The action of the fuzzy logic can be seen in the handover portion (red line), when the normalized bandwidth degraded to 0.08 at flow acceptance number 8,11 , and 13 the fuzzy logic adjusts the bandwidth so that more $\mathrm{HO}$ traffics can be accommodated.

\section{Conclusion}

In this paper, we presented a partition-based call admission control using fuzzy logic controller (FZ CAC). The 


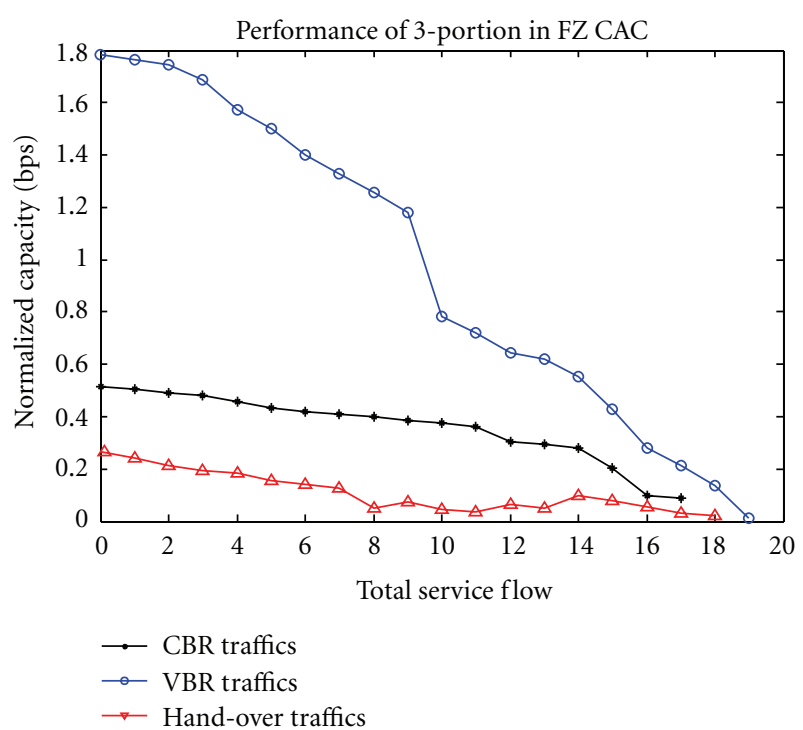

FIGURE 13: Normalized capacity of the total acceptance flow in 3 portions of FZ CAC.

performance of the proposed approach is compared to $\mathrm{PB}$ $\mathrm{CAC}$ and $\mathrm{CB}$ CAC. The results showed that the proposed approach outperformed the aforementioned approaches in terms of total acceptance flow and low dropping probability. FZ CAC has a lower blocking probability than CB CAC. The developed scheme provides better QoS in maintaining mobility for service flow in the network due to low dropping probability.

\section{Acknowledgments}

The authors would like to thank all those who contributed toward making this research successful. Also, we would like to thank all the reviewers for their insightful comment. This work was sponsored by the Research Management Unit, Universiti Teknologi Malaysia.

\section{References}

[1] WiMAX Forum, K. Ramadas, and R. Jain, "WiMAX System Evaluation Methodology," version 2.1, July 2008.

[2] IEEE Std. 802.16e, "IEEE standard for local and metropolitan area networks, part 16: air interface for fixed and mobile broadband wireless access systems, amendment 2: physical and medium access control layers for combined fixed and mobile operation in licensed band and corrigendum 1," May 2005.

[3] G. Chu, D. Wang, and S. Mei, "A QoS architecture for the MAC protocol of IEEE 802.16 BWA system," in Proceedings of the IEEE International Conference on Communications, Circuits and Systems and West Sino Expositions, Chengdu, China, June 2002.

[4] M. Hawa and D. Petr, "Quality of service scheduling in cable and broadband wireless access systems," in Proceedings of the 10th International Workshop on Quality of Service, Miami Beach, Fla, USA, May 2002.
[5] J. Chen, W. Jiao, and H. Wang, "A service flow management strategy for IEEE 802.16 broadband wireless access systems in TDD mode," in Proceedings of the IEEE International Conference on Communications (ICC '05), pp. 3422-3426, Seoul, Republic of Korea, May 2005.

[6] O. E. Falowo and H. A. Chan, "Adaptive bandwidth management and joint call admission control to enhance system utilization and QoS in heterogeneous wireless networks," EURASIP Journal on Wireless Communications and Networking, vol. 2007, Article ID 34378, 2007.

[7] S. Mopati and D. Sarkar, "Call admission control in mobile cellular system, using fuzzy associated memory," in Proceedings of the 12th International Conference on Computer Communications and Networks (ICCCN'03), pp. 95-100, October 2003.

[8] C.-J. Huang, Y.-T. Chuang, D.-X. Yang, I.-F. Chen, Y.-J. Chen, and K. W. Hu, "A mobility-aware link enhancement mechanism for vehicular Ad Hoc networks," EURASIP Journal on Wireless Communications and Networking, vol. 2008, Article ID 862456, 2008.

[9] S. Chandra and A. Sahoo, "An efficient call admission control for IEEE802.16 networks," in Proceedings of the 15th IEEE Workshop on Local and Metropolitan Area Networks, pp. 188193, Princeton, NJ, USA, June 2007.

[10] D. S. Shu'aibu, S. K. Syed-Yusof, and N. Fisal, "Partition-base bandwidth managements for mobile WiMAX IEEE802.16e," International Review on Computers and Software, vol. 5, no. 4, pp. 445-452, 2010.

[11] B. Sklar, Digital Communications: Fundamentals and Application, Prentice-Hall, Upper Saddle River, NJ, USA, 2nd edition, 2001.

[12] K. Hirota, Industrial Applications of Fuzzy Technology, Springer, New York, NY, USA, 1993.

[13] M. Tim Jones, AI Application Programming, Charles River Media, Hingham, Mass, USA, 2nd edition, 2005. 

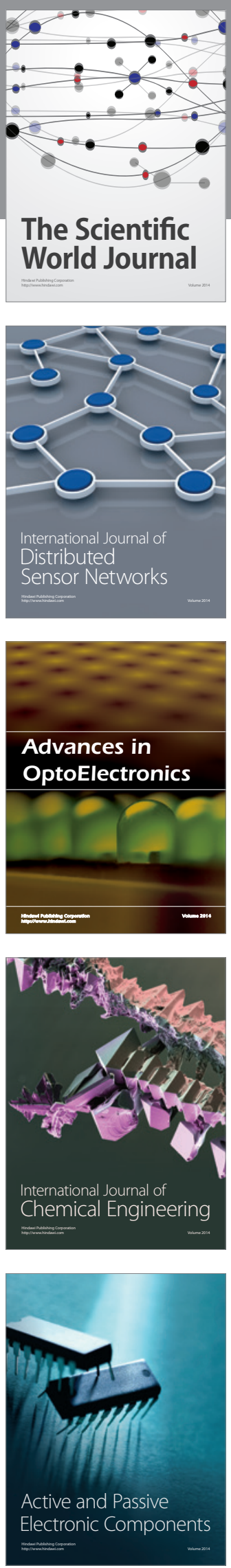
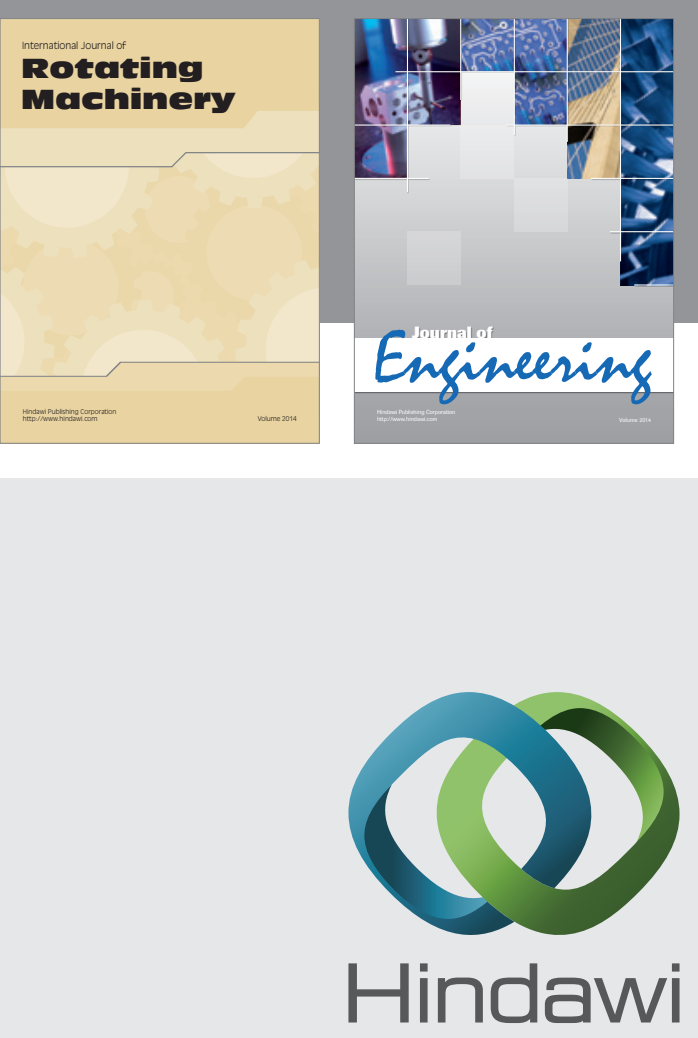

Submit your manuscripts at

http://www.hindawi.com
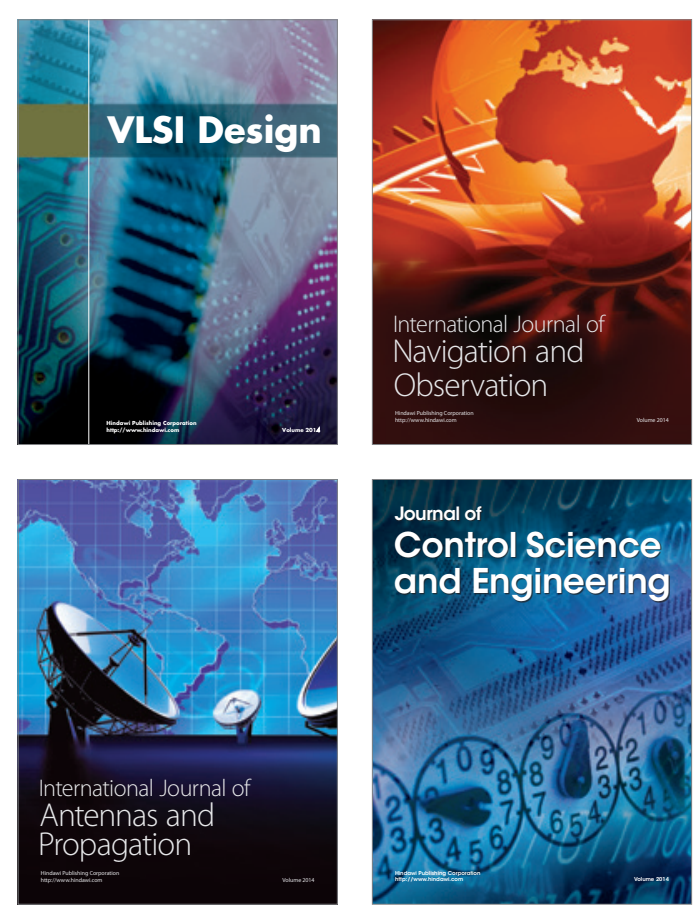
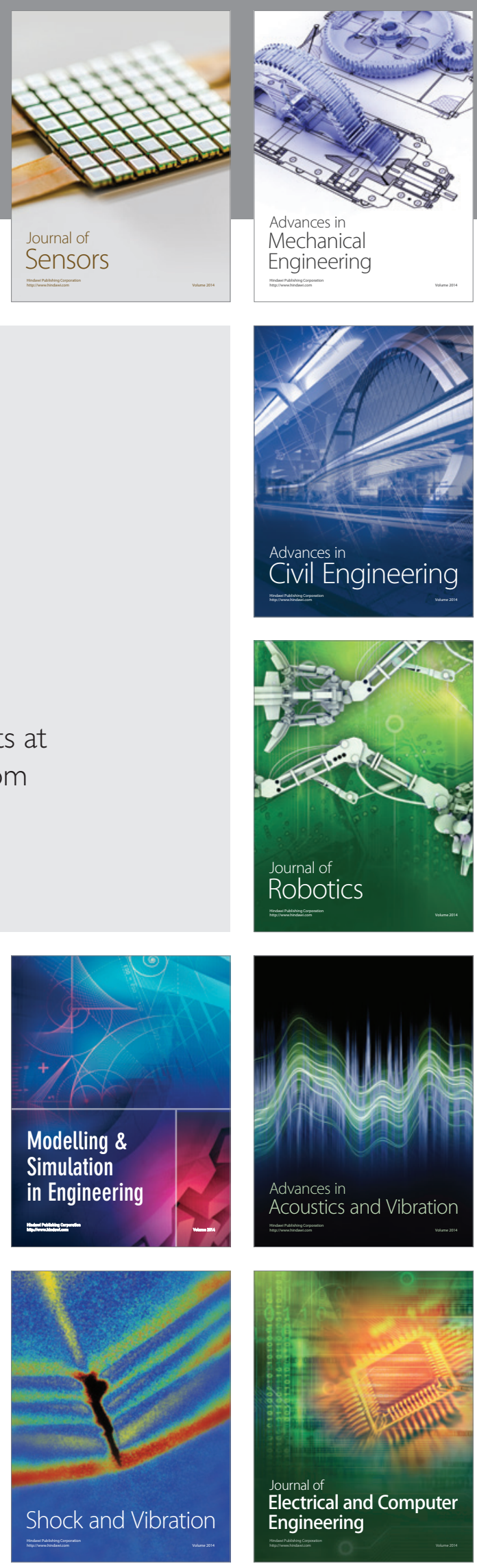